\title{
Resistance exercise performance variability at submaximal intensities in older and younger adults
}

This article was published in the following Dove Press journal:

Clinical Interventions in Aging

17 January 2014

Number of times this article has been viewed

\author{
Gregory J Grosicki' \\ Michael E Miller ${ }^{2}$ \\ Anthony P Marsh' \\ 'Department of Health and \\ Exercise Science, Wake Forest \\ University, Winston-Salem, NC, USA; \\ ${ }^{2}$ Department of Biostatistical Sciences, \\ Division of Public Health Sciences, \\ Wake Forest University School of \\ Medicine, Winston-Salem, NC, USA
}

Correspondence: Anthony P Marsh

Wake Forest University, Department

of Health and Exercise Science, PO Box

7868, Winston-Salem, NC 27I09, USA

$\mathrm{Tel}+\mathrm{I} 3367584643$

Fax +I 3367584680

Email marshap@wfu.edu

\begin{abstract}
We assessed the variability in the number of repetitions completed at submaximal loads in three resistance tasks in older $(\mathrm{N}=32,16$ female, $74.3 \pm 5.4$ years $)$ and younger $(\mathrm{N}=16$, 8 female, $22.8 \pm 1.8$ years) men and women. One repetition maximum (1RM) was determined on two separate visits on three tasks: leg press (LP), leg extension (LE), and bicep curl (BC). Subjects then completed repetitions to failure on each of the three tasks during two visits, a minimum of 48 hours apart, at either 60\% 1RM or $80 \% 1 \mathrm{RM}$. High reliability for all 1RM assessments was observed. Greater muscular strength was observed in younger compared to older men and women on all tasks $(P<0.05)$. At both $60 \%$ and $80 \% 1 \mathrm{RM}$, considerable interindividual variability was observed in the number of repetitions completed. However, the average number of repetitions completed by younger and older men and women at $60 \%$ and $80 \% 1 \mathrm{RM}$ in each of the three tasks was similar, with the only significant difference occurring between younger and older men at $80 \% 1 \mathrm{RM}$ on the leg press $(P=0.0258)$. We did not observe any abnormal blood pressure responses to either the 1RM testing or maximal repetition testing sessions. Considerable interindividual variability was observed in the number of repetitions completed by younger and older men and women at relative intensities typical of resistance training programs. Practitioners should give consideration to individual variability when attempting to maximize the benefits of resistance training.
\end{abstract}

Keywords: resistance exercise, exercise prescription, relative intensity, reliability, older adults, blood pressure

\section{Introduction}

By 2050, approximately 90 million Americans will be $\geq 65$ years of age. ${ }^{1}$ It is well documented that older adults have an increased likelihood of developing significant health issues that limit independence, particularly chronic diseases and disability. ${ }^{2}$ As the number of older adults in the US rises, a critical public health priority will be the identification and implementation of countermeasures designed to attenuate the decline of physical function and the onset of disability in older people.

Sarcopenia, the steady decline of lean muscle mass seen with advancing age, is a significant threat to an older person's ability to remain healthy and function independently. ${ }^{3}$ Decreases in muscle mass are associated with a decline in muscle strength. ${ }^{4}$ Resistance training is an effective countermeasure for sarcopenia and it improves muscle strength and physical function in older adults. ${ }^{3,5,6}$ As a result of the compelling evidence from resistance training studies in older individuals showing favorable effects on muscle and physical function, ${ }^{5,7,8}$ recent American College of Sports Medicine (ACSM) position stands have increased emphasis on resistance training for older adults. ${ }^{9}$ 
Resistance training prescription is frequently based on a percentage of an individual's one repetition maximum (1RM), defined as the maximum amount of weight that can be lifted with proper technique for only one repetition for a specific exercise. ${ }^{10}$ The National Strength and Conditioning Association (NSCA) has published a chart that indicates the number of repetitions theoretically possible at a range of submaximal percentages of $1 \mathrm{RM} .{ }^{10}$ This chart appears to be based on data from previous studies in younger adults. ${ }^{1-17}$ These studies indicate that more repetitions can be completed at lower percentages of $1 \mathrm{RM}^{18-20}$ and more repetitions will be completed on large-muscle mass, multi-joint tasks such as the leg press, than smaller muscle mass, single-joint tasks such as the bicep curl. ${ }^{20-22}$ We are not aware of any published studies that have examined the variability in the number of repetitions completed at submaximal intensities in older men and women. This is an important issue as resistance exercise is an integral component of a well-rounded activity program for older adults.

The purpose of this study was to determine if there was significant interindividual variability in the capacity to lift submaximal loads in older and younger men and women in both multi- and single-joint tasks. This is a necessary first step before proceeding to studies designed to examine mechanisms that might explain interindividual variability in submaximal lifting capacity. An important part of this investigation was the reliable assessment of 1RM in both younger and older adults on the leg press, leg extension, and bicep curl. We hypothesized that: more repetitions will be completed on the same task at $60 \%$ compared to $80 \% 1 \mathrm{RM}$; at $60 \%$ or $80 \% 1 \mathrm{RM}$, more repetitions will be completed on the leg press compared to the bicep curl; and at $60 \%$ or $80 \%$ $1 \mathrm{RM}$, younger adults will complete more repetitions compared to older adults on each task. We also recorded blood pressure responses to maximal effort testing.

\section{Methods}

\section{Experimental approach to the problem}

Subjects completed four assessment visits, on four separate days, with a minimum of 48 hours between each visit. In the first two visits, we determined the subject's one repetition maximum (1RM) for three tasks: leg press, leg extension, and bicep curl. We chose these tasks to include a largemuscle mass multi-joint task (leg press), a large-muscle mass single-joint task (leg extension), and a smaller muscle-mass single-joint task (bicep curl). At the third visit, subjects were instructed to complete repetitions to failure on each task using either $60 \%$ or $80 \% 1 \mathrm{RM}$. At the fourth visit, repetitions to failure were repeated at the other submaximal intensity. The percentages of 1RM were chosen to encompass typical recommendations for resistance training programs. ${ }^{23}$ We used the Cybex 16150 plate-loaded leg press machine, the Cybex 5230 plate-loaded leg extension machine, and Cap Barbell's 4-pound, 1-inch diameter standard E-Z curl bar (RB-47T) for the bicep curl.

\section{Subjects}

We recruited 32 older adults (74.3 \pm 5.4 years, 16 female) from a community-based chronic disease management program and 16 younger adults ( $22.8 \pm 1.8$ years, 8 female) from the Wake Forest University campus. Previous studies have examined variability in repetitions in younger adults using a comparable sample size, ${ }^{11-17}$ but to our knowledge, this is the first study to address this issue in older adults. Therefore, we increased our sample size of older adults to protect against Type II error. Resistance training and 1RM testing experience were assessed by self-report. Subject characteristics are reported in Table 1. Subjects were excluded from the study if they reported any musculoskeletal, visual, auditory, orthopedic, or neuromuscular deficit that might interfere with proper lifting technique or that might be exacerbated as a result of the study protocol. Subjects with a history of unstable angina, congestive heart failure, or exercise-induced complex ventricular arrhythmias were also excluded. This study was approved by the Institutional Review Board at Wake Forest University and all subjects provided written informed consent.

\section{Procedures}

\section{Visit I: consent/data collection and familiarization}

At visit 1 we obtained written informed consent, collected each subject's anthropometric data and assessed, by selfreport, their resistance training and 1RM testing experience. Each subject was then familiarized with the three tasks. Subjects then completed 1RM testing as described below.

\section{Visit I and 2: one repetition maximum testing}

One repetition maximum was determined according to procedures described by Kraemer and Fry. ${ }^{24}$ The initial warm-up load on visit 1 was subjectively determined by the study investigator, a trained exercise physiologist, who used subject height, body mass, and training experience as a guide. Loads for subsequent warm-up sets were established using ratings of perceived exertion (Borg 10-point scale) a technique added by the study investigators to the established protocol to quantitatively measure subject exertion during previous warm-up sets. 
Table I Subject characteristics and blood pressure response

\begin{tabular}{|c|c|c|c|c|}
\hline & \multicolumn{2}{|l|}{ Men } & \multicolumn{2}{|l|}{ Women } \\
\hline & Younger, $n=8$ & Older, $n=16$ & Younger, $n=8$ & Older, $n=16$ \\
\hline Age & $23.5 \pm 1.6$ & $73.6 \pm 6.1$ & $22.0 \pm 1.9$ & $75.1 \pm 4.5$ \\
\hline Height (cm) & $183.3 \pm 5.6^{\mathrm{a}}$ & $174.2 \pm 6.9$ & $166.8 \pm 6.8$ & $163.0 \pm 5.5$ \\
\hline Body mass (kg) & $81.4 \pm 8.1$ & $85.5 \pm 10.5$ & $61.8 \pm 10.4$ & $72.3 \pm 13.2^{b}$ \\
\hline Resistance training experience & 6 of 8 & 13 of 16 & 5 of 8 & 14 of 16 \\
\hline IRM experience & 4 of 8 & 3 of 16 & 4 of 8 & I of 16 \\
\hline \multicolumn{5}{|l|}{ Blood pressure response } \\
\hline Visit 3 systolic pre & $124.8 \pm 13.9$ & $121.3 \pm 12.8$ & $112.5 \pm 6.1$ & $121.6 \pm 12.3^{c}$ \\
\hline Visit 3 systolic post & $127.3 \pm 9.4$ & $120.4 \pm|5|$. & $109.9 \pm 40.6$ & $119.5 \pm 11.7$ \\
\hline Visit 3 diastolic pre & $72.8 \pm 6.8$ & $69.9 \pm 6.4$ & $66.8 \pm 7.5$ & $72.6 \pm 7.0^{\mathrm{d}}$ \\
\hline Visit 3 diastolic post & $70.0 \pm 7.2$ & $67.8 \pm 5.7$ & $66.5 \pm 8.5$ & $68.5 \pm 6.5$ \\
\hline
\end{tabular}

Notes: Values are mean \pm SD. aSignificant difference compared to corresponding value in older men; ${ }^{b}$ significant difference compared to corresponding value in younger women; 'significant difference compared to corresponding value in younger women; 'significant difference compared to corresponding post-assessment blood pressure. Abbreviations: RM, repetition maximum; SD, standard deviation.

Each 1RM attempt was separated by $3-5$ minutes of rest. The $1 \mathrm{RM}$ testing was conducted for each of the three tasks on the same day with a 20 -minute break between tasks. The bicep curl task separated the leg press and the leg extension, and the order of the leg press and leg extension tasks was randomized. For visit 2, the subject returned for another series of 1RM testing at least 48 hours later (average time between visit 1 and $2=7.3 \pm 5.4$ days). The task order established at visit 1 was also used during visit 2 .

\section{Visit 3 and 4: maximal repetition testing}

The maximal repetition sessions involved completing repetitions to failure for each of the three tasks, using the order established during 1RM testing. Half of the subjects were assigned $60 \% 1 \mathrm{RM}$ on visit 3 and $80 \% 1 \mathrm{RM}$ on visit 4 , and the other half were assigned the opposite order. Resting blood pressure was measured prior to beginning each visit and 3-5 minutes after completing each assessment session. No subject was permitted to begin an assessment session or leave the testing facility after the session with a blood pressure greater than 160/100 mmHg. The blood pressure measurements for visit 3 are shown in Table 1. Following blood pressure measurements, subjects were asked to complete a 5-minute unloaded warm-up on a Monark Ergomedic 8-18E exercise bicycle followed by a light warm-up set of five repetitions using $50 \%$ of their 1RM for each task. Ninety seconds after the warm-up, subjects were asked to complete repetitions to failure at either $60 \%$ or $80 \% 1 \mathrm{RM}$ on the assigned task.

During the maximal repetition efforts, the investigator closely observed each repetition and only counted efforts that were completed with proper form through the full range of motion. Subjects were encouraged to complete repetitions consecutively. Verbal encouragement was standardized using a script during all testing sessions. ${ }^{25,26}$ The movement speed was controlled using a metronome allowing 2 seconds for the concentric phase and 2 seconds for the eccentric phase of the task. Testing was stopped if a subject could no longer keep time with the metronome. Subjects were given 20 minutes of rest between each task. There was a minimum of 48 hours between visits. Average time between visits 2 and 3 was $4.9 \pm 2.4$ days and between visits 3 and 4, $6.9 \pm 5.7$ days. The variability of the time between visits $1-2$, $2-3$, and 3-4 was due to the logistics of accommodating each subject's schedule.

\section{Statistical analyses}

Statistical analyses of demographic data were performed using SPSS (v19.0; IBM Corporation, Armonk, NY, USA). Statistical significance was set at $P<0.05$ level for each test. Due to the large number of hypotheses being tested, we were willing to accept the possibility of making a Type I error in exchange for identifying relationships to be studied more thoroughly in future studies. Statistical analyses of 1RM and repetition data were performed using SAS (v9.3; SAS Institute Inc., Cary, NC, USA).

\section{Descriptive statistics}

Descriptive statistics of baseline data included means and standard deviations of age, height, body mass, and blood pressure response. We used a Student's $t$-test for independent samples to check for baseline differences between younger and older men and women.

\section{IRM data}

Test-retest reliability of 1RM tests was examined using the intraclass correlation coefficient (ICC-one way 
random model) using the maximal weight lifted on each task at visits 1 and 2. Mixed effects analyses of variance using restricted maximum likelihood estimation were used to analyze 1RM task outcome data (LP, LE, and BC) within sex. Each model contained a main effect for visit (visit 1 or visit 2), age (young adults or old adults), and a visit by age interaction. The covariance structure for the repeated measures allowed for separate estimates of the between and within person variability (variance components) for each age group. Contrasts were used to test for equality of means between visits and young versus old for visit 2 . Then, models assuming common variance components (eg, the same between person variance component for each age group) were fitted and likelihood ratio tests were used to test for equality of variances. We note that due to the small sample sizes within sex ( $\mathrm{N}=8$ for young adults; $\mathrm{N}=16$ for older adults), these tests have limited power to detect small differences.

\section{Repetition data}

Analysis of the number of repetitions performed at $60 \%$ and $80 \%$ of $1 \mathrm{RM}$ for each task was performed separately for each task and within sex/age groups. We used paired $t$-tests to assess equality of the mean number of repetitions at $60 \%$ versus $80 \%$ of 1 RM. Pitman's test for equality of variances in dependent samples ${ }^{27}$ was used to test for equality of variances at $60 \%$ versus $80 \%$ of 1RM within sex/age group; whereas, an $F$-test for equality of variances was used to test for variance equality between young and old adults at $60 \%$ and $80 \%$ of 1 RM, within sex.

A mixed models two-way analysis of variance (ANOVA) was used to compare the mean number of repetitions completed by task and age, separately for men and women. In the case that no significant interaction of age and task was identified, the interaction term was dropped from the model, and a test of main effects was run to identify any potential effect of either age or task on the number of repetitions completed. In the case that a significant $(P<0.05)$ task by age interaction was observed, multiple pairwise comparisons were used to compare the mean number of repetitions between groups defined by the cross-classification of age and task.

\section{Results}

All subjects (total of 32 older and 16 younger adults) completed all aspects of the study. The demographic data of our sample are presented in Table 1.

Younger men were significantly taller $(P<0.003)$, but there was no difference in body mass $(P=0.304)$, training experience $(P=0.750)$, or $1 \mathrm{RM}$ experience $(P=0.172)$ compared to older men. Compared to younger women, older women had more body mass $(P<0.047)$ but there was no difference in height $(P=0.205)$, training experience $(P=0.244)$, or $1 \mathrm{RM}$ experience $(P=0.057)$.

There was no difference in systolic or diastolic blood pressure between younger and older men and women during visit 3, except older women had a higher pre-assessment systolic blood pressure compared to younger women $(P=0.024)$. There was no significant change in blood pressure pre-versus post-assessment, except in older women where a significant decrease in diastolic blood pressure was observed $(P=0.015)$. No adverse events occurred during any of the 192 (48 subjects $\times 4$ sessions per subject) 1 RM or maximal repetition testing sessions in older or younger adults.

\section{IRM testing}

Reliability analyses of the 1RM testing in both older and younger women and older and younger men are presented in Table 2. Intraclass correlation coefficients (ICC) for 1RM testing were $0.990,0.910$, and 0.900 in older women, and $0.970,0.970$, and 0.930 in younger women on the leg press, leg extension, and bicep curl, respectively. ICC coefficients in men were similar, with values of $0.980,0.980$, and 0.960 in older men, and 0.990, 0.940, and 0.980 in younger men. An ANOVA between visit 1 and visit 2 of 1RM testing is presented in Table 2. In all four groups, there was a significant increase $(P<0.05)$ in $1 \mathrm{RM}$ measures on the second testing visit for each task, except in younger men on the bicep curl $(P=0.053)$.

For each of the three tasks, younger men and women lifted significantly more weight on visit 2 of $1 \mathrm{RM}$ testing than older men and women (Table 2, $P<0.001 ; P<0.01$ respectively). Although not tested formally, inspection of Table 2 suggests that there is greater between person variance for the leg press compared to either the leg extension or bicep curl in both younger and older men and women. The within person variance also appears to be greater for the leg press compared to the other two tasks in younger and older women and older men.

\section{Repetition data}

The variability in the number of repetitions completed at the two task intensities in each of the three tasks is illustrated in Figure 1 using side-by-side frequency histograms. These histograms illustrate repetition frequency for each of the three tasks at $60 \%$ and $80 \% 1 \mathrm{RM}$ in older and younger adults. On the $\mathrm{x}$-axis is the number of people to complete a given number of repetitions, and on the y-axis is the number 
Table 2 Analysis of women's and men's IRM data

\begin{tabular}{|c|c|c|c|c|}
\hline Task & $\begin{array}{l}\text { Day I } \\
\text { Mean (SD) } 95 \% \text { CI }\end{array}$ & $\begin{array}{l}\text { Day } 2 \\
\text { Mean (SD) } 95 \% \mathrm{Cl}\end{array}$ & $\begin{array}{l}\text { Between person } \\
\text { variance }\end{array}$ & $\begin{array}{l}\text { Within person } \\
\text { variance }\end{array}$ \\
\hline \multicolumn{5}{|l|}{ Women } \\
\hline \multicolumn{5}{|l|}{ Young $(\mathrm{N}=8)$} \\
\hline Leg press $(\mathrm{kg})$ & $244.7(83.86)(183.2-306.1)$ & $262.2^{\mathrm{a}, \mathrm{b}}(76.62)(206.1-3 \mid 8.4)$ & $6,233.8($ ICC $=0.97)$ & 218.05 \\
\hline Leg extension $(\mathrm{kg})$ & $57.0(14.16)(46.6-67.4)$ & $59.5^{\mathrm{a}, \mathrm{b}}(15.32)(48.3-70.8)$ & $212.2(\mathrm{ICC}=0.97)$ & 5.47 \\
\hline Bicep curl (kg) & I9.I (4.53) (I5.9-22.4) & $20.5^{\mathrm{a}, \mathrm{b}}(4.33)(17.4-23.7)$ & I8.2 (ICC $=0.93)$ & 1.42 \\
\hline \multicolumn{5}{|l|}{ Old $(\mathrm{N}=16)$} \\
\hline Leg press (kg) & $90.1(57.28)(60.4-119.8)$ & $96.2^{\mathrm{a}}(54.34)(68.1-124.4)$ & $3,110.1 \quad(I C C=0.99)$ & 16.97 \\
\hline Leg extension $(\mathrm{kg})$ & $27.4(7.6 I)(23.4-31.3)$ & $29.4^{a}(8.39)(25.1-33.8)$ & $58.5(I C C=0.91)$ & 5.68 \\
\hline Bicep curl (kg) & $13.0(3.46)(11.2-14.8)$ & $14.1^{\mathrm{a}}(3.79)(12.2-16.1)$ & $11.8($ ICC $=0.90)$ & 1.37 \\
\hline \multicolumn{5}{|l|}{ Men } \\
\hline \multicolumn{5}{|l|}{ Young $(\mathrm{N}=8)$} \\
\hline Leg press $(\mathrm{kg})$ & 347.3 (80.39) (288.3-406.2) & $358 . I^{c, d}(85.7 I)(295.2-420.9)$ & $6,872.0(\mathrm{ICC}=0.99)$ & 32.15 \\
\hline Leg extension $(\mathrm{kg})$ & $101.5(27.24)(81.5-121.5)$ & II0.0 $0^{c, d}(24.22)(92.2-127.8)$ & $625.3($ ICC $=0.94)$ & 38.76 \\
\hline Bicep curl (kg) & $40.4(6.06)(35.9-44.8)$ & $41.2^{\mathrm{d}}(5.28)(37.4-45.1)$ & $31.6(I C C=0.98)$ & 0.69 \\
\hline \multicolumn{5}{|l|}{ Old $(\mathrm{N}=16)$} \\
\hline Leg press $(\mathrm{kg})$ & 167.5 (69.59) (I31.5-203.6) & $\mid 76.8^{c}(70.75)(|40|-2 \mid 3.4)$. & $4,858.0(\mathrm{ICC}=0.98)$ & 79.37 \\
\hline Leg extension $(\mathrm{kg})$ & $61.2(14.77)(53.6-68.9)$ & $63.5^{c}(13.28)(56.6-70.4)$ & I $93.7($ ICC $=0.98)$ & 3.43 \\
\hline Bicep curl (kg) & $25.1(5.14)(22.4-27.7)$ & $26.1^{\mathrm{c}}(5.15)(23.4-28.7)$ & $25.5(\mathrm{ICC}=0.96)$ & 1.02 \\
\hline
\end{tabular}

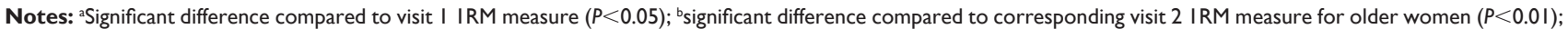
'significant difference compared to visit I IRM measure $(P<0.05)$; dsignificant difference compared to corresponding visit 2 IRM measure for older men $(P<0.01)$. Abbreviations: $\mathrm{Cl}$, confidence interval; ICC, intraclass correlation coefficient; RM, repetition maximum; SD, standard deviation.

of repetitions completed. At both $60 \%$ and $80 \% 1 \mathrm{RM}$, considerable interindividual variability was observed in the number of repetitions completed by older and younger men and women. However, the average number of repetitions completed by older and younger men and women at $60 \%$ and $80 \% 1 \mathrm{RM}$ in each of the three tasks was remarkably similar, with the only significant difference occurring in younger and older men at $80 \% 1 \mathrm{RM}$ on the leg press $(P=0.0258$, Table 3$)$. A paired $t$-test of equal means (Table 3 ) showed that both older and younger men and women completed significantly more repetitions at $60 \% 1 \mathrm{RM}$ than at $80 \% 1 \mathrm{RM}$ on each of the three tasks $(P<0.05)$.

A test of equal variances was used to compare the variance observed at $60 \%$ versus $80 \% 1 \mathrm{RM}$ for each of the three tasks in older and younger men and women (Table 3 ). In older women, greater variance was observed at $60 \%$ $1 \mathrm{RM}$ than $80 \% 1 \mathrm{RM}$ on the leg press and the bicep curl $(P<0.001)$. In younger women, no significant difference in variance was observed at $60 \%$ versus $80 \% 1 \mathrm{RM}$ in any of the three tasks. In older men, there was a significant difference in variance between $60 \%$ and $80 \% 1 \mathrm{RM}$ on the leg press $(P<0.05)$. In younger men, a significant difference in variance was found between $60 \%$ and $80 \% 1 \mathrm{RM}$ in all three tasks $(P<0.05)$.

Tests of equal variance between older and younger adults were done separately for men and women to check for differences in variability between age groups (Table 3 ).
At $60 \% 1 \mathrm{RM}$ on the bicep curl, greater variability was observed in older women than younger women $(P<0.001)$. However, once an outlier was removed (an older woman who was stopped after completing 100 repetitions at $60 \%$ 1RM), no significant difference in variability between the groups was observed $(P=0.120)$. No significant differences in variability were observed at $60 \%$ or $80 \% 1 \mathrm{RM}$ in older and younger women on the leg press and leg extension (Table 3). When comparing older and younger men, no significant differences in the variability of the number of repetitions completed on the leg extension or the bicep curl at either intensity were found (Table 3). However, on the leg press, more variability was observed in the number of repetitions completed by younger adults at both $60 \%(P=0.016)$ and $80 \% 1 \mathrm{RM}(P=0.007)$.

A mixed models ANOVA was used to determine the effect of the task on the number of repetitions that older and younger men and women were able to complete at $60 \%$ and $80 \% 1 \mathrm{RM}$ (Table 4). At 60\% 1RM, the task had a significant effect on the number of repetitions completed by both men $(P=0.003)$ and women $(P=0.001)$. At $60 \% 1 \mathrm{RM}$, a greater number of repetitions were completed by men and women on the leg press compared to the bicep curl (men: $P=0.0060$; women: $P=0.0001$ ) or leg extension (men: $P=0.0010$; women: $P=0.0048)$. At $60 \% 1 \mathrm{RM}$, women completed a greater number of repetitions on the bicep curl than the leg extension ( $P=0.0108)$, but no difference was observed in the 

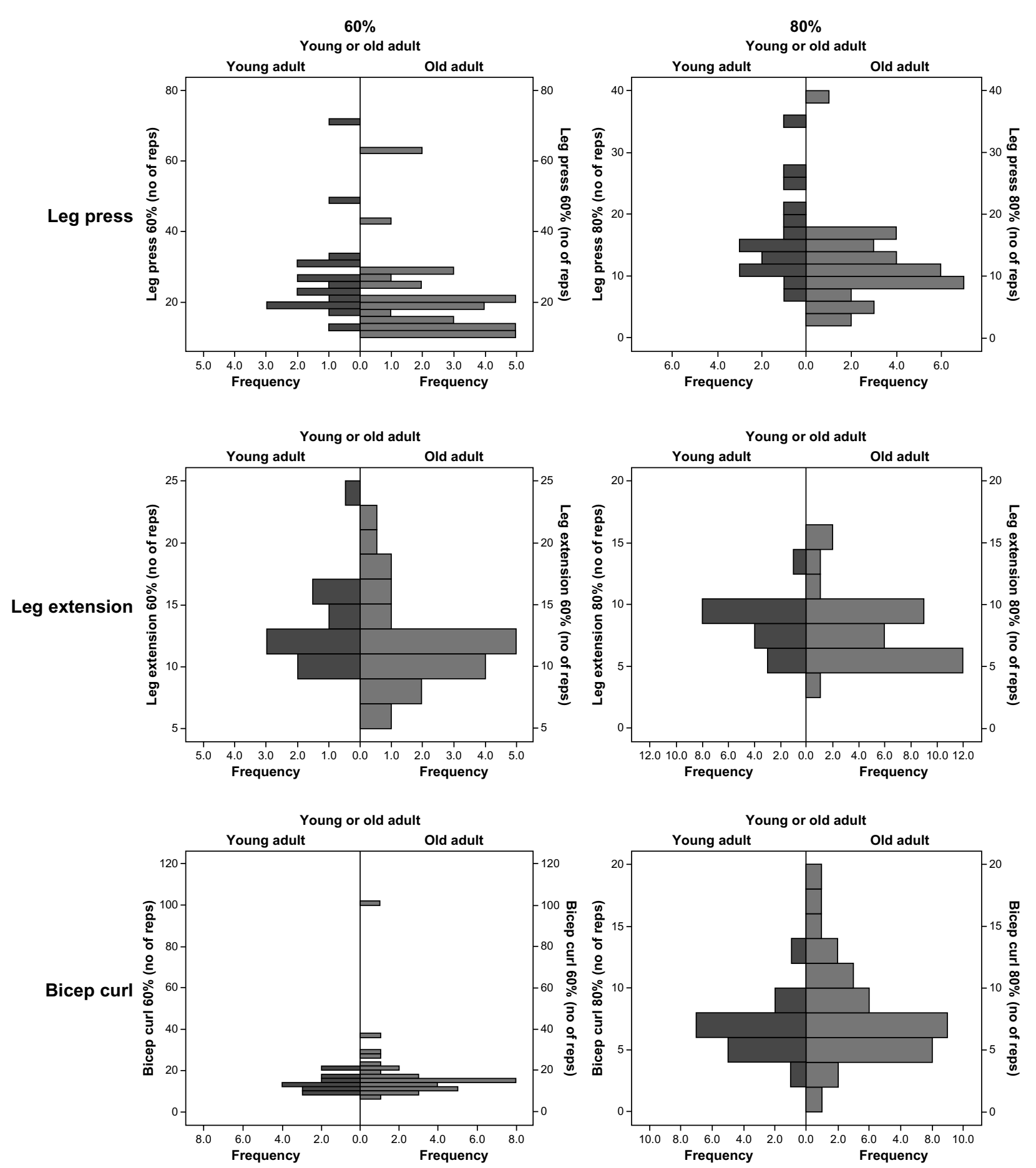

Figure I Variability in submaximal IRM intensities on leg press, leg extension, and bicep curl.

Notes: Histograms illustrate repetition frequency for each of the three tasks at $60 \%$ and $80 \%$ IRM in older and younger adults. On the $\mathrm{x}$-axis is the number of people to complete a given number of repetitions, and on the $y$-axis is the number of repetitions completed.

Abbreviations: RM, repetition maximum; reps, repetitions; no, number.

number of repetitions completed by men on bicep curl and leg extension $(P=0.2620)$.

At $80 \% 1 \mathrm{RM}$, the task had a significant impact on the number of repetitions that women were able to complete ( $P=0.0017)$. Women completed a greater number of repetitions at $80 \% 1 \mathrm{RM}$ on the leg press than the bicep curl
( $P=0.0006)$ or the leg extension $(P=0.0010)$. Student's $t$-tests comparing the number of repetitions by task at $80 \%$ 1RM (Table 3) demonstrated that younger men were able to complete a greater number of repetitions on the leg press than either the bicep curl $(P=0.0002)$ or the leg extension $(P=0.0005)$. In older men at $80 \% 1 \mathrm{RM}$, significantly more 
Table 3 Number of repetitions completed at $60 \%$ and $80 \%$ IRM in women and men

\begin{tabular}{|c|c|c|c|c|}
\hline Task & $\begin{array}{l}60 \% \text { IRM } \\
\text { Mean (SD) } 95 \% \mathrm{CI}\end{array}$ & $\begin{array}{l}80 \% \text { IRM } \\
\text { Mean (SD) } 95 \% \text { CI }\end{array}$ & $\begin{array}{l}P \text {-value for paired } \\
t \text {-test of equal means }\end{array}$ & $\begin{array}{l}P \text {-value for test of } \\
\text { equal variances }^{\mathrm{a}}\end{array}$ \\
\hline \multicolumn{5}{|l|}{ Women } \\
\hline \multicolumn{5}{|l|}{ Young $(\mathrm{N}=8)$} \\
\hline Leg press & $28.8(9.21)(22.0-35.5)$ & $17.0(6.57)(|2.2-2| .8)$ & $<0.001$ & 0.165 \\
\hline Leg extension & II.4 (2.33) (9.7-13.1) & $8.8(1.98)(7.3-10.2)$ & $<0.001$ & 0.508 \\
\hline Bicep curl & $13.0(4.34)^{b}(9.8-16.2)$ & $6.5(3.16)(4.2-8.8)$ & $<0.001$ & 0.313 \\
\hline \multicolumn{5}{|l|}{ Old $(\mathrm{N}=16)$} \\
\hline Leg press & $23.3(16.37)(\mid 4.8-31.7)$ & I2.8 (7.83) (8.7-|6.8) & 0.002 & $<0.001$ \\
\hline Leg extension & II.I (4.15) (9.0-13.3) & $7.9(3.40)(6.1-9.6)$ & $<0.001$ & 0.296 \\
\hline Bicep curl & $21.3(22.32)(9.7-32.9)$ & $8.9(4.33)(6.7-11.2)$ & 0.023 & $<0.001$ \\
\hline $\begin{array}{l}\text { Bicep curl (Remove } \\
\text { outlier with } 100 \text { reps) }\end{array}$ & I6.I (7.87) (|2.4-2I.4) & $8.9(4.33)(6.7-11.2)$ & $<0.001$ & 0.002 \\
\hline Task & $\begin{array}{l}60 \% \text { IRM } \\
\text { Mean (SD) } 95 \% \mathrm{Cl}\end{array}$ & $\begin{array}{l}80 \% \text { IRM } \\
\text { Mean (SD) } 95 \% \text { CI }\end{array}$ & $\begin{array}{l}P \text {-value for paired } \\
t \text {-test of equal means }\end{array}$ & $\begin{array}{l}P \text {-value for test of } \\
\text { equal variances }^{\mathrm{a}}\end{array}$ \\
\hline \multicolumn{5}{|l|}{ Men } \\
\hline \multicolumn{5}{|l|}{ Young $(\mathrm{N}=8)$} \\
\hline Leg press & $26.3(18.20)^{c}(12.9-39.6)$ & $\mid 4.9^{\mathrm{d}, \mathrm{g}}(8.37)^{\mathrm{c}}(8.7-2 \mid .0)$ & 0.018 & $<0.001$ \\
\hline Leg extension & I3.9 (4.36) (I0.7-I7.I) & $7.3(1.98)(5.8-8.7)$ & 0.001 & 0.037 \\
\hline Bicep curl ${ }^{\mathrm{a}}$ & $12.9(3.98)(10.0-15.8)$ & $6.1(1.55)(5.0-7.3)$ & 0.001 & 0.024 \\
\hline \multicolumn{5}{|l|}{ Old $(\mathrm{N}=16)$} \\
\hline Leg press & I9.3 (8.69) (I4.7-23.8) & $9.1^{f}(3.68)(7.2-11.0)$ & $<0.001$ & 0.003 \\
\hline Leg extension & II.8 (3.07) (I0.2-13.3) & $8.6^{\mathrm{e}}(2.66)(7.2-9.9)$ & $<0.001$ & 0.531 \\
\hline Bicep curl ${ }^{\mathrm{a}}$ & I4.3 (4.78) (II.8-I6.8) & $6.1(2.98)(4.5-7.6)$ & $<0.001$ & 0.084 \\
\hline
\end{tabular}

Notes: aPittman's test for equality of variances in dependent samples; 'bignificant difference compared to corresponding variance in older women ( $<<0.00 \mathrm{I})$; 'significant difference compared to corresponding variance in older men $(P<0.05)$; ${ }^{d}$ significant difference compared to corresponding value in younger men on bicep curl $(P=0.0002)$ and leg extension ( $P=0.0005)$; 'significant difference compared to corresponding value in older men on bicep curl ( $P=0.0124)$; f ignificant difference compared to corresponding

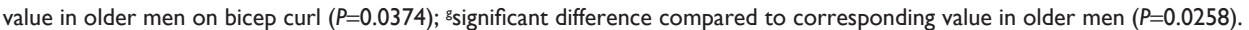

Abbreviations: $\mathrm{Cl}$, confidence interval; $\mathrm{RM}$, repetition maximum; SD, standard deviation.

repetitions were completed on the leg extension $(P=0.0124)$ and leg press $(P=0.0374)$ compared to the bicep curl.

At $60 \% 1 \mathrm{RM}$, no effect of age on the number of repetitions that men and women were able to complete was observed. Similarly, at $80 \% 1 \mathrm{RM}$ in women, age had no effect on the number of repetitions completed. At $80 \% 1 \mathrm{RM}$, a mixed model ANOVA (Table 4) demonstrated a significant interaction between task and age on the number of repetitions that men were able to complete. Although no difference for age was observed at $80 \% 1 \mathrm{RM}$ on the leg extension or bicep curl, younger men completed a greater number of repetitions on the leg press compared to older men $(P=0.0258)$.

\section{Discussion}

To our knowledge, this is the first study to examine, in detail, the interindividual variability in the number of repetitions completed by older men and women at submaximal percentages of $1 \mathrm{RM}$ for several common resistance

Table 4 Mixed models ANOVA for repetitions

\begin{tabular}{|c|c|c|c|c|}
\hline \multirow[t]{2}{*}{$\%$ IRM } & \multicolumn{2}{|l|}{ Women } & \multicolumn{2}{|l|}{ Men } \\
\hline & 60 & 80 & 60 & 80 \\
\hline Test of task by & $P=0.13$ & $P=0.06$ & $P=0.19$ & $P=0.02^{a}$ \\
\hline age interaction & $P=0.30^{*}$ & & & \\
\hline Main effect for task* & $P=0.00 I^{*, b}$ & $P=0.0017^{b}$ & $P=0.003^{b}$ & See Table 3 above for \\
\hline Main effect for age* & $P=0.83^{*}$ & $P=0.7303$ & $P=0.72$ & comparisons (notes $\mathrm{d}-\mathrm{g}$ ) \\
\hline Compare LP to BC & $P=0.000 I^{*, c}$ & $P=0.0006^{c}$ & $P=0.0060^{c}$ & \\
\hline Compare LP to LE & $P=0.0048^{*, c}$ & $P=0.0010^{c}$ & $P=0.0010^{c}$ & \\
\hline Compare BC to LE & $P=0.0108^{*, c}$ & $P=0.97$ & $P=0.2620$ & \\
\hline
\end{tabular}

Notes: Unstructured covariance because of large heterogeneity of variance across tasks; *remove bicep curl outlier; andicates significant task by age interaction; btest of simple main effects for task demonstrate significant effect of task; 'indicates significant difference in number of repetitions completed between tasks.

Abbreviations: BC, bicep curl; LE, leg extension; LP, leg press; RM, repetition maximum; ANOVA, analysis of variance. 
training exercises. Previous studies have reported variability in repetitions in younger and middle-aged adults. ${ }^{18-20}$ Of note, we did not observe a single instance of abnormal BP response to either the 1RM testing or maximal repetition testing sessions. Because older subjects were recruited from a community-based chronic disease management program, the majority were accustomed to regular physical activity. Although many of the older subjects reported past cardiac events or that they were suffering from arthritis, there were no adverse events and no complaints that joint pain was aggravated by the assessments. Our observations are consistent with those of Gordon et al who reviewed the safety of dynamic maximal strength testing in older adults and did not identify a single cardiovascular event in over 26,000 assessment sessions..$^{28}$

An important aspect of this study was the identification of each subject's 1RM. For this reason, we used a familiarization session and 1RM was measured for each of the three tasks on two separate visits, using the detailed protocol described by Kraemer and Fry. ${ }^{24}$ Previously, Phillips et al reported that $1 \mathrm{RM}$ could be determined in older adults with high confidence after three familiarization sessions and two to three testing visits. ${ }^{29}$ We achieved high 1RM reliability as is demonstrated by the high intraclass correlation coefficients produced for 1RM in both age groups (Table 2) using only a single familiarization session and two testing visits. Pragmatically, implementing a protocol that required three familiarization sessions followed by two to three testing sessions in a large randomized trial or longitudinal epidemiological study would be challenging. The protocol we used of one familiarization session and two $1 \mathrm{RM}$ testing visits to determine maximal strength provides high reliability and is a more feasible alternative.

In spite of the high reliability achieved during 1RM testing, we observed a large range and considerable interindividual variability in the number of repetitions completed by both older and younger men and women for a given task at $60 \%$ and $80 \%$ of $1 \mathrm{RM}$. Our data have implications for practitioners and exercise interventionists engaged in prescribing resistance training programs and highlight the potential pitfall of using tables or formulas that are designed to provide guidance on the number of repetitions achievable at selected percentages of $1 \mathrm{RM}$. For example, at $80 \% 1 \mathrm{RM}$, the table by the National Strength and Conditioning Association indicates that eight repetitions are expected..$^{10}$ At $80 \% 1 \mathrm{RM}$ on the leg press, older adults completed 11 (range: 2-38) repetitions, while younger adults in our study completed a mean of 16 (range: 6-34) repetitions. The variability in the number of repetitions completed at $80 \% 1 \mathrm{RM}$ demonstrates the challenges of prescribing resistance exercise based on a percentage of an individual's maximum.

The number of repetitions completed at a given percent of $1 \mathrm{RM}$ varied depending on the task (Table 4). At $60 \% 1 \mathrm{RM}$, both older and younger men and women completed a greater number of repetitions on the leg press, a large-muscle mass multi-joint task, than the bicep curl (smaller muscle mass single-joint task) or the leg extension (large-muscle mass single-joint task). In younger trained adults, Shimano et al observed that at $60 \% 1 \mathrm{RM}$, subjects were able to complete a greater number of repetitions on the back squat (29.9 \pm 7.4 ), a large-muscle mass multi-joint task, than on the arm curl (19.0 \pm 2.9 ), a smaller muscle mass single-joint task. ${ }^{20}$ Also in younger adults, Hoeger et al ${ }^{18,19}$ found that subjects were able to complete significantly more repetitions on the leg press than on seven other machinebased exercises involving smaller muscle mass. Because larger muscles have a greater number of motor units, it has been proposed that the alternating contraction of these motor units, a process known as asynchronous recruitment, enables these muscles to repeat submaximal contractions for a longer period of time. ${ }^{20,30}$

At $80 \%$ 1RM, both younger and older adults completed a greater number of repetitions on the leg press than the bicep curl. Otherwise, differences between the number of repetitions completed between tasks at $80 \% 1 \mathrm{RM}$ were less pronounced compared to $60 \%$ (Table 3 ). This finding is consistent with previous research in younger subjects by both Hoeger et $\mathrm{a}^{19}$ and Shimano et $\mathrm{al}^{20}$ where differences across tasks for the number of repetitions that subjects were able to complete were shown to decrease with increasing intensity. For example, at 40\% 1RM in younger trained males, Hoeger et a ${ }^{19}$ observed differences across six of seven different tasks. At 80\% 1RM, differences were only observed across three tasks.

Although pronounced differences in muscular strength were observed in older and younger men and women, age had no effect on the number of repetitions that subjects could complete (Table 4), except in men at $80 \%$ of $1 \mathrm{RM}$ on the leg press (Table 3). In a comparison of muscular strength and endurance during constant velocity knee extension on a dynamometer, LaForest et $\mathrm{al}^{31}$ observed a significant decline in peak torque between younger and older active men and women, especially at higher speeds $(P<0.001)$, but no age difference was observed in muscular endurance. A number of other studies have also observed age-related differences in muscular strength and peak torque but not endurance. ${ }^{32-34}$ 
A challenge to interpretation of this previous research comparing muscular endurance in different populations is the use of different measures to assess muscular endurance. While some studies have measured muscular endurance as a decline in torque or velocity over repeated constant velocity contractions, ${ }^{6,33,35}$ others have quantified it as the maximal number of repetitions an individual can complete at a selected percentage of 1RM. ${ }^{18-20,22}$

In a study comparing 1RM, concentric knee extensor peak power, and fatigability in 28 younger and 24 older men and women, Petrella et $\mathrm{al}^{35}$ observed that older adults were less capable of sustaining maximum concentric velocity during repetitive contractions $(P<0.05)$. Data on muscle mass and aging suggest that age-associated muscle wasting is more likely to produce deficits in muscular power and peak torque, and less likely to affect muscular endurance. $^{31-35}$

The results from this study are best interpreted in the context of the study's strengths and limitations. First, although our sample was of modest size (32 older subjects, 16 younger subjects), it was comparable in number to or greater than previous studies. A strength of our study was the use of a detailed and scripted 1RM protocol at each 1RM testing visit. The maximal repetition testing also followed a detailed protocol to reduce the potential for experimenter bias. The majority of our sample was well-educated white individuals, most of whom reported prior resistance training experience. Future investigations should include minorities and less active individuals. We used a rigorous 1RM protocol described by Kraemer and Fry. ${ }^{24}$ However, the initial warm-up load for subjects was subjectively determined by the study investigator, a trained exercise physiologist, who used subject height, body mass, and training experience as a guide. Loads for subsequent warm-up sets on visit 1 were established with ratings of perceived exertion using the Borg 10 -point scale, a technique added by the study investigators to the established protocol as a means of measuring subject exertion during previous warm-up sets. It is possible that an initial warm-up load that was either too heavy or too light might have decreased accuracy of 1RM measures on visit 1 by asking participants to either over or under exert themselves, inadequately preparing them to lift a maximal load. To control for this, on the second visit for 1RM testing, warm-up loads were established objectively, using percentages of 1RM measures from visit 1 . We provided subjects with one familiarization session and two 1RM testing sessions. Our data support the use of at least two 1RM testing sessions, as higher 1RM's were achieved for each of the three tasks on the second visit of 1RM testing, except for younger men on the bicep curl (Table 2). Without a second measurement of 1RM, subjects would have been completing repetitions to failure with percentages less than their true $60 \%$ or $80 \% 1 \mathrm{RM}$ values, likely increasing the number of repetitions completed.

\section{Practical applications}

Our data demonstrate that the number of repetitions completed by older and younger men and women at submaximal relative intensities can vary substantially. For example, at $80 \% 1 \mathrm{RM}$, the number of repetitions completed by our subjects ranged from 1-38 repetitions. The NSCA table indicates that eight repetitions are expected at $80 \% 1 \mathrm{RM} .{ }^{10}$ Consider the impact of an exercise interventionist prescribing eight repetitions at $80 \% 1 \mathrm{RM}$ to subjects in our study. While some individuals would find eight repetitions far too easy, others would find it impossible. Also, the nature of the task (single versus multi-joint) and the amount of muscle mass involved will influence the number of repetitions an individual will be able to complete at a given percentage of 1RM. One practical approach used by skilled exercise interventionists that addresses the issue of variability is to ask an individual to complete a maximal number of repetitions in the final set for a particular exercise. The weight lifted can be adjusted so that the number of repetitions possible is limited to a narrow range (10-12 reps). Subsequently, training load is increased when a subject exceeds the upper end of the range on two consecutive training sessions. Regardless of the preferred protocol, exercise interventionists should consider interindividual variability when attempting to maximize the benefits of resistance exercise and understand the potential for large differences in individual performance and responses to resistance training protocols even when relative intensity is established using well-accepted methods. Clinicians can take solace in knowing that $1 \mathrm{RM}$ testing in older adults, with characteristics consistent with those assessed in this study, does not appear to produce any abnormal cardiovascular responses or lead to musculoskeletal injury.

\section{Acknowledgments}

We thank Dr Peter Brubaker for comments on an early draft of this manuscript. Dr Marsh and Dr Miller are supported by the WFU Claude D Pepper Center National Institutes for Aging P30 AG021332. Funding provided by a Science Research Fund grant from the Office of Research and Sponsored Programs at Wake Forest University. 


\section{Disclosure}

The authors report no conflicts of interest in this work.

\section{References}

1. Vincent G, Velkoff V. The Next Four Decades: The Older Population in the United States 2010 to 2050. In: US Department of Commerce EaSA, ed 2013:25-1138.

2. Guralnik JM, Simonsick EM. Physical disability in older Americans. J Gerontol. 1993;48 Spec No:3-10.

3. Evans WJ. Exercise and nutritional needs of elderly people: effects on muscle and bone. Gerodontology. 1998;15(1):15-24.

4. Roth SM, Ferrell RE, Hurley BF. Strength training for the prevention and treatment of sarcopenia: sarcopenia in aging. J Nutr Health Aging. 2000;4(3):143-155.

5. Fiatarone MA, O'Neill EF, Ryan ND, et al. Exercise training and nutritional supplementation for physical frailty in very elderly people. N Engl J Med. 1994;330(25):1769-1775.

6. Latham NK, Bennett DA, Stretton CM, Anderson CS. Systematic review of progressive resistance strength training in older adults. $J$ Gerontol A Biol Sci Med Sci. 2004;59(1):48-61.

7. Brown AB, McCartney N, Sale DG. Positive adaptations to weightlifting training in the elderly. J Appl Physiol (1985). 1990;69(5): $1725-1733$.

8. Hunter GR, McCarthy JP, Bamman MM. Effects of resistance training on older adults. Sports Med. 2004;34(5):329-348.

9. Garber CE, Blissmer B, Deschenes MR, et al; American College of Sports Medicine. American College of Sports Medicine position stand. Quantity and quality of exercise for developing and maintaining cardiorespiratory, musculoskeletal, and neuromotor fitness in apparently healthy adults: guidance for prescribing exercise. Med Sci Sports Exerc. 2011;43(7):1334-1359.

10. Earl R, Baechle T. NSCA's Essentials of Personal Training. Champaign, IL: Human Kinetics; 2003.

11. Epley B. Poundage Chart: Boyd-Epley Workout. Lincoln, NE: Body Enterprises; 1985.

12. Brzycki M. Strength testing: predicting a one rep max from reps to fatigue. J Health Phys Ed Rec Dance. 1993;64:88-90.

13. Wathen D. Load assignment. Essentials of Strength and Conditioning. Champaign, IL: Human Kinetics; 1994:435-446.

14. Baechle T, Earl R. Fitness and Weight Training. Champaign, IL: Human Kinetics; 1995.

15. Brzycki M. Assessing strength. Fitness Manage. 2000:34-37.

16. Baechle T, Earl R. Resistance Training: Essentials of Strength and Conditioning. Champaign, IL: Human Kinetics; 2008.

17. Bompa T, Haff G. Periodization: Theory and Methodology of Training. Champaign, IL: Human Kinetics; 2009.

18. Hoeger WW, Barette SL, Hale DF, Hopkins DR. Relationship between repetitions and selected percentages of one repetition maximum. J Strength Cond Res. 1987;1(1):11-13.
19. Hoeger W, Hopkins DR, Barr SI, Hale S. Relationship between repetitions and selected percentages of one repetition maximum. A comparison between untrained and trained males and females. J Appl Sport Sci Res. 1990:47-54.

20. Shimano T, Kraemer WJ, Spiering BA, et al. Relationship between the number of repetitions and selected percentages of one repetition maximum in free weight exercises in trained and untrained men. $J$ Strength Cond Res. 2006;20(4):819-823.

21. Faigenbaum A, Westcott W, Long C, Loud R, Delmonico M, Micheli M. Relationship between repetitions and selected percentages of the one repetition maximum in healthy children. Pediatr J Phys Ther. 1998;10: 110-113.

22. da Cunha Nascimento D, Prestes J, Tibana RA, Balsamo S. Comparison of the number of repetitions and perceived exertion between multi-joint and single-joint exercise at different intensities in untrained women. Braz J Biomotricity. 2011;5(2):96-105.

23. Fleck S, Kraemer W. Designing Resistance Training Programs. Champaign, IL: Human Kinetics; 2004.

24. Kraemer W, Fry A. Development and Evaluation of Methodology. Champaign, IL: Human Kinetics; 1995.

25. Mazzetti SA, Kraemer WJ, Volek JS, et al. The influence of direct supervision of resistance training on strength performance. Med Sci Sports Exerc. 2000;32(6):1175-1184.

26. Wise JB, Posner AE, Walker GL. Verbal messages strengthen bench press efficacy. J Strength Cond Res. 2004;18(1):26-29.

27. Pitman E. A note on normal correlation. Biometrika. 1939;31(1-2): $9-12$.

28. Gordon NF, Kohl HW, Pollock ML, Vaandrager H, Gibbons LW, Blair SN. Cardiovascular safety of maximal strength testing in healthy adults. Am J Cardiol. 1995;76(11):851-853.

29. Phillips WT, Batterham AM, Valenzuela JE, Burkett LN. Reliability of maximal strength testing in older adults. Arch Phys Med Rehabil. 2004;85(2):329-334.

30. McDonnall D, Clark GA, Normann RA. Selective motor unit recruitment via intrafascicular multielectrode stimulation. Can J Physiol Pharmacol. 2004;82(8-9):599-609.

31. Laforest S, St-Pierre DM, Cyr J, Gayton D. Effects of age and regular exercise on muscle strength and endurance. Eur J Appl Physiol Occup Physiol. 1990;60(2):104-111.

32. Aniansson A, Grimby G, Hedberg M, Rungren A, Sperling L. Muscle function in old age. Scand J Rehabil Med Suppl. 1978;6:43-49.

33. Larsson L, Grimby G, Karlsson J. Muscle strength and speed of movement in relation to age and muscle morphology. $J$ Appl Physiol Respir Environ Exerc Physiol. 1979;46(3):451-456.

34. Lindström B, Lexell J, Gerdle B, Downham D. Skeletal muscle fatigue and endurance in young and old men and women. $J$ Gerontol A Biol Sci Med Sci. 1997;52(1):B59-B66.

35. Petrella JK. Age differences in knee extension power, contractile velocity, and fatigability. J Appl Physiol. 2004;98(1):211-220.
Clinical Interventions in Aging

\section{Publish your work in this journal}

Clinical Interventions in Aging is an international, peer-reviewed journal focusing on evidence-based reports on the value or lack thereof of treatments intended to prevent or delay the onset of maladaptive correlates of aging in human beings. This journal is indexed on PubMed Central, MedLine, the American Chemical Society's 'Chemical Abstracts
Dovepress

Service' (CAS), Scopus and the Elsevier Bibliographic databases. The manuscript management system is completely online and includes a very quick and fair peer-review system, which is all easy to use. Visit http://www.dovepress.com/testimonials.php to read real quotes from published authors. 